Stoecker, Holger, Afrikawissenschaften in Berlin von 1919 bis 1945. Zur Geschichte und Topographie eines wissenschaftlichen Netzwerkes

\title{
Chantal Metzger
}

\section{OpenEdition}

\section{Journals}

Édition électronique

URL : http://journals.openedition.org/ifha/2256

DOI : 10.4000/ifha.2256

ISSN : 2198-8943

Éditeur

IFRA - Institut franco-allemand (sciences historiques et sociales)

Référence électronique

Chantal Metzger, « Stoecker, Holger, Afrikawissenschaften in Berlin von 1919 bis 1945. Zur Geschichte und Topographie eines wissenschaftlichen Netzwerkes », Revue de l'IFHA [En ligne], Date de recension, mis en ligne le 01 janvier 2010, consulté le 22 septembre 2020. URL : http://journals.openedition.org/ifha/ 2256 ; DOI : https://doi.org/10.4000/ifha.2256

Ce document a été généré automatiquement le 22 septembre 2020.

(CIFHA 


\title{
Stoecker, Holger,
} Afrikawissenschaften in Berlin von 1919 bis 1945. Zur Geschichte und Topographie eines wissenschaftlichen Netzwerkes

\author{
Chantal Metzger
}

1 Cet ouvrage représente la version abrégée de la thèse de H.S. : Afrikawissenschaften in Berlin von 1919 bis 1945, présentée en 2006 devant la Humboldt-Universität de Berlin. Il s'agit un travail original qui ne se contente pas de reprendre, voire de compléter les travaux existant sur les africanistes. Il est pionnier à plus d'un titre car pour la première fois, un historien, avec les outils des historiens, étudie l'action des africanistes sur une longue période en couvrant la République de Weimar et la totalité du Troisième Reich. H.S. s'intéresse tout particulièrement aux relations existant entre scientifiques et pouvoir politique et contrairement à Hans Fischer, il estime qu'il y a eu une forme de partenariat entre scientifiques et Troisième Reich.

2 L'auteur définit tout d'abord son champ de recherche. Le terme d' " africaniste » a été employé pour la première fois par le linguiste Carl Meinhof en 1914 et concerne au départ les spécialistes des langues et de la littérature africaines. Dans l'Entre-deuxguerres, cette définition s'élargit aux spécialistes de la linguistique, de l'ethnologie et aux travaux d'historiens sur l'Afrique sub-saharienne. C'est Dietrich Westermann qui lui donne son sens le plus moderne, proche de la définition anglaise : l'africaniste devient un expert de l'Afrique. H.S. s'interroge dans sa thèse sur les arguments développés par d'africanistes et leurs partenaires au sein des lobbies coloniaux et des milieux d'affaires de 1919 à 1945. Son étude s'articule autour de quatre axes principaux : les relations entre la théorie et l'innovation : jusqu'à quel point existait-il avant la Première Guerre mondiale une recherche sur l'Afrique, quelle était la place de Berlin dans le débat national et international autour de ces recherches ? ; l'étude des 
africanistes en tant que société scientifique, groupe de spécialistes au sein du monde scientifique ; les rapports entre le monde politique (et notamment le ministre de la Culture de Prusse Carl Heinrich Becker) et ces africanistes mais aussi l'évolution de ces rapports sous le Troisième Reich (contact avec le Kolonialpolitisches Amt der NSDAP et avec les associations coloniales) ; les organismes, instituts, chaires d'université chargées de ces recherches.

3 Les africanistes viennent de diverses disciplines dans les universités de Berlin et de Hambourg mais surtout des sciences humaines et sociales. Parmi eux figurent des linguistes, des ethnologues, mais aussi des musicologues, des juristes, des anthropologues, des géologues, des spécialistes de l'art, de l'histoire et des religions d'outre-mer. Certains d'entre eux sont orientalistes, missionnaires, linguistes, historiens, conservateurs de musées, de bibliothèques et souvent membres des associations coloniales. Le réseau constitué par le monde des africanistes présente la particularité d'être un réseau scientifique mais qui dépend du monde politique et qui s'inscrit dans une perspective de recherches internationales. Selon H.S., la période de l'Entre-deux-guerres fut la plus fructueuse pour cette communauté scientifique qui s'était professionnalisée, en fait, dès les années vingt.

Les africanistes s'étaient déjà manifestés pendant la Première Guerre mondiale. Le linguiste Carl Meinhof profite de la présence de prisonniers africains dans les camps pour étudier langues et dialectes, une commission enregistre, sous la direction de Doegen, les voix de ces prisonniers et ces enregistrements sont remis à la Preussische Staatsbibliothek de Berlin, nouvellement créée. La société africaine, ses langues, sa culture ont été, dès l'époque impériale, un des thèmes les plus étudiés à l'Université de Berlin sur laquelle l'auteur concentre son étude en négligeant un peu trop Hambourg où le Kolonialinstitut, fondé en 1908, a déjà, en 1912, vingt-deux professeurs titulaires et quarante-quatre assistants - et Tübingen. La capitale du Reich attire bien sûr les plus importants africanistes. D. Westermann s'y rend dès 1905 et il y obtient, en 1921, la chaire de langues africaine.

5 La perte des colonies à Versailles, sans désorienter les africanistes allemands, a néanmoins des répercussions sur leurs recherches. Elle relance paradoxalement l'intérêt pour les études africaines. Les gouvernements de la République de Weimar entendent maintenir une chaire de langue africaine pour les missionnaires, les hommes d'affaires et les techniciens (p. 66) qui envisagent de se rendre dans ces territoires. Les cours attirent également des lecteurs étrangers. C'est en 1920 qu'est créée une Société de secours destinée aux chercheurs (Notgemeinschaft), qui prendra le nom de Deutsche Forschungsgemeinschaft en 1935. Elle est présidée, à ses débuts, par l'ancien ministre prussien des cultes F. Schmidt-Ott, et se transforme progressivement sous le Troisième Reich en organe de planification de la recherche scientifique. H.S. en a exploité minutieusement les archives et a constaté que les africanistes y participent activement et obtiennent l'aide de cette société.

6 Sous la République de Weimar se constitue donc une véritable communauté scientifique d'africanistes dont $\mathrm{D}$. Westermann est le chef de file. Elle s'engage dans la lutte contre « le mensonge colonial » qui visait à faire des colons allemands de mauvais gestionnaires de leurs colonies. Les africanistes constituent d'excellents agents du révisionnisme colonial sous la République de Weimar. Mais comme l'Allemagne n'a plus d'empire colonial, certains d'entre eux, à l'instar de Moritz Julius Bonn, ont une autre attitude, cet africaniste suggérant une « décolonisation » générale. Dans ce contexte, 
l'arrivée au pouvoir d'Hitler en 1933 ne constitue pas, selon H.S., une césure. Les africanistes de Berlin ne font aucune déclaration de loyauté au nouveau régime, certains d'entre eux émigrent et ne reviendront pas après la guerre. Ceux qui restent, comme Westermann, sont séduits par la perspective d'une révision du traité de Versailles et du retour des anciennes colonies. On a indéniablement, comme l'écrit l'auteur, une symbiose entre le monde des africanistes et la politique menée par les partisans - des conservateurs wilhelminiens - du retour des colonies. H.S. pense que cet intérêt se tarit en 1936-1938 (p. 108), ce qui semble surprenant au moment où le Reichskolonialbund et le Kolonialpolitischesamt der NSDAP se lancent dans une active campagne en faveur du retour des colonies à l'Allemagne ! La décision de former des fonctionnaires destinés à encadrer un futur empire colonial (administration, armée, police, médecine) a pour effet bien au contraire d'augmenter les auditeurs, mais il ne s'agit plus de volontaires, ni de personnes motivées par des études dans le domaine de l'ethnologie ou des langues africaines. Nombreux seront les africanistes, qui, à l'instar de Westermann, participent, en 1940, activement à l'élaboration des grands projets reconstituant une Mittelafrika allemande. Tous ces rêves de colonisation allemande s'effondrent en janvier 1943 avant même le départ du dernier soldat allemand de Tunisie et les activités des africanistes se réduisent considérablement. H.S. évoque enfin, dans sa conclusion, le sort réservé après guerre à certains africanistes comme Martin Heepe ou Westermann et l'évolution de la recherche sur l'Afrique dans les deux États allemands après leur création.

7 Cette contribution qui couvre l'Entre-deux-guerres et la Seconde Guerre mondiale est des plus précieuses. Au carrefour de l'histoire des sciences, de la société et des idées, elle touche aussi aux relations internationales entre savants en temps de paix et de guerre. Il s'agit donc d'un travail neuf, original, d'un grand intérêt historique. En attestent l'ampleur des recherches, la pertinence et la finesse des analyses et de la démonstration. Il mérite une suite pour l'après-guerre.

Chantal Metzger (Université Nancy 2) 